\title{
Super-roughening versus intrinsic anomalous scaling of surfaces
}

\author{
Juan M. López ${ }^{1}$, Miguel A. Rodríguez ${ }^{2}$, and Rodolfo Cuerno ${ }^{3}$ \\ ${ }^{1}$ Department of Mathematics, Imperial College, 180 Queen's Gate, London SW7 2BZ, United Kingdom \\ ${ }^{2}$ Instituto de Física de Cantabria, Consejo Superior de Investigaciones Científicas - Universidad de Cantabria, \\ Avenida de los Castros s/n, E-39005 Santander, Spain \\ ${ }^{3}$ Departamento de Matemáticas y Grupo Interdisciplinar de Sistemas Complicados \\ Universidad Carlos III de Madrid, c/ Butarque 15, E-28911 Leganés, Spain
}

\begin{abstract}
In this paper we study kinetically rough surfaces which display anomalous scaling in their local properties such as roughness, or height-height correlation function. By studying the power spectrum of the surface and its relation to the height-height correlation, we distinguish two independent causes for anomalous scaling. One is super-roughening (global roughness exponent larger than or equal to one), even if the spectrum behaves non anomalously. Another cause is what we term an intrinsically anomalous spectrum, in whose scaling an independent exponent exists, which induces different scaling properties for small and large length scales (that is, the surface is not self-affine). In this case, the surface does not need to be super-rough in order to display anomalous scaling. In both cases, we show how to extract the independent exponents and scaling relations from the correlation functions, and we illustrate our analysis with two exactly solvable examples. One is the simplest linear equation for molecular beam epitaxy, well known to display anomalous scaling due to superroughening. The second example is a random diffusion equation, which features anomalous scaling independent of the value of the global roughness exponent below or above one.
\end{abstract}

PACS numbers: 5.40.+j, 05.70.Ln, 68.35.Fx

\section{INTRODUCTION}

The problem of the dynamics of growing surfaces has attracted very much attention in recent years. A large variety of discrete models and continuous equations have been investigated numerically and analytically in order to explain the diversity of rough surfaces observed in nonequilibrium natural processes [1] In many cases, it is observed that the surface morphology displays time and space fluctuations which have scaling properties akin to those of equilibrium critical phenomena. Most of the work is thus devoted to identifying the different universality classes to which the models studied belong, which are basically determined by the exponents characterizing the fluctuations of the interface.

We will be interested in this work in the dynamics of a one dimensional surface, which will be described by the stochastic variable $h(x, t)$ giving the height of the surface at time $t$ above substrate position $x$. The physical properties of the system are thus described by the moments of $h(x, t)$. For instance, the global width

$$
W(L, t)=\left\langle\left\langle(h(x, t)-\bar{h}(t))^{2}\right\rangle_{x}^{1 / 2}\right\rangle,
$$

is a measure of the interface fluctuations. In (11), $\langle\ldots\rangle_{x}$ denotes spatial average over the whole system of lateral size $L, \bar{h}(t) \equiv\langle h(x, t)\rangle_{x}$ is the average value of the height at time $t$, and $\langle\ldots\rangle$ is an average over realizations of the noise. Starting from a initially flat surface, the global width is observed to satisfy in many cases the dynamic scaling Ansatz of Family-Vicsek [5],

$$
W(L, t)=t^{\chi / z} f\left(L / t^{1 / z}\right),
$$

where the scaling function $f(u)$ behaves as

$$
f(u) \sim\left\{\begin{array}{ll}
\text { const. } & \text { if } u \gg 1 \\
u^{\chi} & \text { if } u \ll 1
\end{array} .\right.
$$

The roughness exponent $\chi$ characterizes the surface morphology in the stationary regime, in which the correlation length $\xi(t) \sim t^{1 / z}$ ( $z$ is the so called dynamic exponent) has reached a value larger than the system size $L$. This happens for times larger than the saturation time, $t \gg t_{s}(L)$, which scales with $L$ as $t_{s}(L) \sim L^{z}$. The ratio $\beta=\chi / z$ is called growth exponent and characterizes the short time behavior of the surface.

The existence of dynamic scaling as in (2), plus the assumption of the self-affine character of the interface - in the sense that there is no characteristic length scale in the surface besides the system size, and therefore all scales obey the same physics - allows one to obtain the exponents from the local width $w(l, t)$, which measures the surface height fluctuations over a window of size $l \ll L$. At times larger than $t_{s}(l) \sim l^{z}$ the local width is thus expected to saturate and

$$
w\left(l, t \gg l^{z}\right) \sim l^{\chi_{l o c}},
$$

where the local roughness exponent $\chi_{l o c}$ should equal the exponent obtained in (2.3), $\chi_{l o c}=\chi$. For short times, $t \ll t_{s}(l)$, the local width scales with time as $w(l, t) \sim t^{\beta}$, which is the short scale analog of the scaling behavior for the global width given in (2). It is an interesting fact to stress here that the equality $\chi_{l o c}=\chi$ in (仼) is not guaranteed in general when Family-Vicsek scaling holds for the 
global width (3), since the self-affinity of the interface is an additional independent condition.

Although the above scaling picture has been successfully applied to a great variety of models, which includes for instance the importat universality classes associated to the Edwards-Wilkinson (EW) [6] and Kardar-ParisiZhang (KPZ) [7] equations, it is not always valid. In very recent studies of discrete models and continuum equations, mainly related with the growth of surfaces by molecular beam epitaxy (MBE), it has been found that in the case of the so-called super-rough surfaces, i.e. for surfaces with a global roughness exponent $\chi>1$, the usual assumption of the equivalence between the global and local descriptions of the surface is not valid. In these systems [8,9] the local width (or equivalently the heightheight correlation function, see below) does not saturate as expected in the standard Family-Vicsek scaling, but crosses over to a new behavior in the intermediate time regime $l^{z} \ll t \ll L^{z}$, characterized by a different growth exponent $\beta_{*}, w\left(l, t \gg l^{z}\right) \sim l t^{\beta_{*}}$, where $\beta_{*}=\beta-1 / z$. It is easy to see that now the local width saturates only when the global width does (this is, $t_{s}(l)=L^{z}$ for all $l$ ) and $w\left(l, t \gg L^{z}\right) \sim l L^{\chi-1}$ in saturation, which, according to (4), yields a local exponent $\chi_{l o c}=1$ for superroughening. This scaling behavior which is not encompassed by the the Family-Vicsek Ansatz has been termed anomalous scaling in the literature [9] 10] and later different models have been studied in which $\chi_{l o c}$ and $\beta_{*}$ take values different from 1 and $\beta-1 / z$, respectively [11].

While it is currently clearly understood that superroughening always leads to anomalous scaling, in a recent paper [12] two of us have demonstrated that growth models in which $\chi<1$ may also exhibit an unconventional scaling behavior with similar scaling relations between exponents. In that reference, for an analytically solvable growth model with tunable values of $\chi$, we identified anomalous dynamic scaling with the lack of self-affinity. Also a class of discrete MBE growth models studied numerically in Ref. [11] behaves anomalously, although the interfaces generated were not super-rough at all. That is, surfaces can display anomalous scaling no matter what their value of the global roughness exponent is, either $\chi>1$ or $\chi<1$. In the presence of anomalous scaling, not all length scales are equivalent in the system; the scaling behavior is different for short (local) and large length scales, hence $\chi_{l o c}$ and $\chi$ differ.

Our aim in the present paper is to complete the picture presented in Ref. [12], showing that the mechanisms which lead to anomalous scaling behavior can be separated into two classes, according to the behavior of the structure factor or power spectrum of the surface, $S(k, t)$, to be defined below. One of the mechanisms is superroughening, even if $S(k, t)$ behaves as in the FamilyVicsek case. The other mechanism corresponds to what we term intrinsic anomalous scaling of the structure factor, where an independent exponent appears which measures the difference between the short and large length scale power laws, namely, the difference between the local and global roughness exponents $\chi_{l o c}$ and $\chi$. We will show how to identify the corresponding anomalous scaling by extracting the independent critical exponents from the correlation functions. As stated above, for simplicity we restric our further analysis to growth models in one dimension, but the conclusions are expected to be valid in arbitrary dimensions. We note that, although the scaling relations to appear below for the super-rough and intrinsic anomalous cases exist already in the literature 10,9], seemingly the independence between the occurrence of anomalous scaling and the value (larger or smaller than one) of the global roughness exponent $\chi$ has not been adequately understood so far. Since a clear picture of the different scaling behaviors which can appear is crucial to correctly identify them in experiments and models, we believe a somewhat detailed presentation is in order. It is attempted in Section II. The rest of the paper is organized as follows. In Section III we illustrate the conclusions of Sec. II by an analysis of two growth models formulated in terms of stochastic equations. Even though the two models are exactly solvable, we choose to study them numerically in order to illustrate how our scaling analysis can be applied to more general cases. The first model examined is the linear MBE equation, known to display anomalous scaling due to super-roughening. The second model we consider in Sec. III is the stochastic diffusion equation with random diffusion coefficient studied in [13, 12], which exemplifies a case of intrinsic anommalous scaling. Finally, we present some conclusions in Sec. IV, and discuss consequences for scaling analysis of rough surfaces in experiments and numerical simulations. Appendix A contains details on the calculations discussed in Sec. II.

\section{ANOMALOUS DYNAMIC SCALING}

In order to study a problem of kinetic roughening the height-height correlation function

$$
G(l, t)=\left\langle\left\langle(h(l+x, t)-h(x, t))^{2}\right\rangle_{x}\right\rangle,
$$

is frequently used. This correlation function scales in the same way as the square of the local width, $G(l, t) \sim$ $w^{2}(l, t)$, and provides an alternative method to determine the critical exponents.

However, as we will see in the following, a more transparent understanding of the complete dynamic scaling can be obtained by studying the Fourier transform of the interface height in a system of linear size $L$ (see $e . g$. [1] and references therein),

$$
\widehat{h}(k, t)=L^{-1 / 2} \sum_{x}[h(x, t)-\bar{h}(t)] \exp (i k x),
$$

where the spatial average of the height has been subtracted. In this representation, the properties of the surface can be investigated by calculating the structure factor or power spectrum 


$$
S(k, t)=\langle\widehat{h}(k, t) \widehat{h}(-k, t)\rangle,
$$

which contains the same information on the system as the height-height correlation function $G(l, t)$ defined in (5), both of them being related by 14.

$$
\begin{aligned}
G(l, t) & =\frac{4}{L} \sum_{2 \pi / L \leq k \leq \pi / a}[1-\cos (k l)] S(k, t) \\
& \propto \int_{2 \pi / L}^{\pi / a} \frac{d k}{2 \pi}[1-\cos (k l)] S(k, t) .
\end{aligned}
$$

\section{A. Family-Vicsek scaling}

Family-Vicsek scaling reads, when expressed in terms of the structure factor,

$$
S(k, t)=k^{-(2 \chi+1)} s_{F V}\left(k t^{1 / z}\right),
$$

with $s_{F V}$ the following scaling function

$$
s_{F V}(u) \sim\left\{\begin{array}{ll}
\text { const. } & \text { if } u \gg 1 \\
u^{2 \chi+1} & \text { if } u \ll 1
\end{array},\right.
$$

Indeed, Eqs. (9,10) can easily be seen to be equivalent to Eqs.(2, 3), by noting that the global width is nothing but the integral of $S(k, t)$, i.e.

$$
W^{2}(L, t)=\frac{1}{L} \sum_{k} S(k, t)=\int \frac{d k}{2 \pi} S(k, t),
$$

where the momentum integral is limited to $2 \pi / L \leq k \leq$ $\pi / a$ and represents a continuum aproximation to the sum over the discrete set of modes. In a discrete growth model, $a$ is identified with the lattice spacing. Note as well that Eq. (10) implies that, for $k t^{1 / z} \gg 1$, the spectrum does not depend on time, and hence at saturation $\left(t^{1 / z} \gg L\right), S(k, t)$ is a pure power law independent of system size.

Going back to real space, and having assumed a Family-Vicsek scaling for $S(k, t)$, Eq. (10), one obtains, using Eq. (8),

$$
\begin{aligned}
G(l, t) & =\int_{\frac{2 \pi}{L}}^{\frac{\pi}{a}}[1-\cos (k l)] \frac{s_{F V}\left(k t^{1 / z}\right)}{k^{2 \chi+1}} d k \\
& =l^{2 \chi} \int_{\frac{2 \pi l}{L}}^{\frac{\pi l}{a}}[1-\cos (u)] \frac{s_{F V}(\rho u)}{u^{2 \chi+1}} d u,
\end{aligned}
$$

where we have introduced the ratio $\rho=t^{1 / z} / l$. The limiting behaviors of (12) are very simple to study when $\chi<1$; the details can be found in the Appendix. For instance, at saturation $t^{1 / z} \gg L$, the scaling function in the integrand of Eq. (12) can be substituted for its (constant) limiting behavior at large arguments (see (10)), and one is left with $G(l, t) \sim l^{2 \chi}$, the limits $a \rightarrow 0$, and
$L \rightarrow \infty$ having been taken. Analogously one can arrive at the local scaling given by

$$
G(l, t) \sim\left\{\begin{array}{ll}
t^{2 \chi / z} & \text { if } t^{1 / z} \ll l \\
l^{2 \chi} & \text { if } t^{1 / z} \gg l
\end{array}\right\}=l^{2 \chi} g_{F V}\left(l / t^{1 / z}\right),
$$

where the scaling function $g_{F V}(u)$ is constant for $u \ll 1$ and $g_{F V}(u) \sim u^{-2 \chi}$ for $u \gg 1$. This is the usual situation in which $\chi<1$ and the local and global roughness exponents are equal $\left(\chi=\chi_{l o c}\right.$ and $\left.\beta_{*} \equiv 0\right)$.

\section{Super-roughening}

In the case of growth models generating super-rough surfaces $(\chi>1)$, but with a structure factor fulfilling Family-Vicsek scaling, the integrals in (12) are divergent in the limit $l \ll t^{1 / z}(\rho \gg 1)$ for $L \rightarrow \infty$, given the strong singularity at the origin of integration [9]. Taking the limit $l \ll t^{1 / z}$ first for fixed $a, L$ (see Appendix), one obtains

$$
G(l, t) \sim\left\{\begin{array}{ll}
l^{2} t^{2(\chi-1) / z} & \text { if } l \ll t^{1 / z} \ll L \\
l^{2} L^{2(\chi-1)} & \text { if } l \ll L \ll t^{1 / z}
\end{array},\right.
$$

so that $\chi_{l o c}=1$ and $\beta_{*}=\beta-1 / z$. In the early time regime $t^{1 / z} \ll l \ll L, G(l, t) \sim t^{2 \chi / z}$. As has been remarked in Refs. [15.16], the fact that $\chi_{l o c}$ cannot exceed 1 for super-rough surfaces $(\chi>1)$ is a purely geometric property which follows from definition (5).

\section{B. Intrinsic anomalous roughening}

Apart from the super-roughening case, there is another important situation which leads to anomalous scaling. In some growth models - an example of which is discussed in Sec. III-, the structure factor may present an unconventional scaling not described by Family-Vicsek (10). Let us consider a dynamic scaling form for $S(k, t)$ as in Eq.(9) but with the scaling function $s_{F V}(u)$ replaced by

$$
s_{A}(u) \sim\left\{\begin{array}{ll}
u^{2 \theta} & \text { if } u \gg 1 \\
u^{2 \chi+1} & \text { if } u \ll 1
\end{array},\right.
$$

where the label $A$ denotes intrinsic anomalous spectrum. Here $\theta$ is a new exponent which "measures" the anomaly in the spectrum. In a system of size $L$, Eqs. (9), (15) hold only up to the saturation time $t_{s}(L)=L^{z}$, after which the system size $L$ replaces the correlation length $t^{1 / z}$ in all expressions. Thus in particular, at saturation the structure factor depends on the size of the system as $S(k, t) \sim L^{\theta} k^{\theta-(2 \chi+1)}$. As a consequence, the stationary spectrum shows severe finite size effects, to the extent that it is not defined in the thermodinamic limit $L \rightarrow \infty$.

A scaling behavior such as Eqs. (9), (15) for the structure factor does not affect the behavior of the global width, which preserves its Family-Vicsek form, $W(L, t) \sim$ $t^{\beta}$ for $t \ll L^{z}$ and $W\left(L, t \gg L^{z}\right) \sim L^{\chi}$. On the contrary, 
the local properties of the surface change dramatically if $S(k, t)$ scales as in (15). That can be seen by computing the height-height correlation function from Eq. (8),

$$
G(l, t)=l^{2 \chi} \int_{\frac{2 \pi l}{L}}^{\frac{\pi l}{a}}[1-\cos (u)] \frac{s_{A}(\rho u)}{u^{2} \chi+1} d u
$$

which as before (and see Appendix) gives $G(l, t) \sim t^{2 \beta}$ for times $t \ll l^{z}$. However, for intermediate times $l^{z} \ll t \ll L^{z}(\rho \gg 1)$ the integral (16) picks up a nontrivial contribution from the behavior of $s_{A}(u)$ at large arguments, so that

$$
G(l, t) \sim l^{2 \chi} \rho^{2 \theta}=l^{2 \chi-2 \theta} t^{2 \theta / z}
$$

Thus, the complete scaling of the height difference correlation function (or, equivalently, the square of the local width) can be written as

$G(l, t) \sim\left\{\begin{array}{ll}t^{2 \beta} & \text { if } t^{1 / z} \ll l \ll L \\ l^{2 \chi_{l o c}} t^{2 \beta_{*}} & \text { if } l \ll t^{1 / z} \ll L \\ l^{2 \chi_{l o c}} L^{2 \theta} & \text { if } l \ll L \ll t^{1 / z}\end{array}\right\}=l^{2 \chi} g_{A}\left(l / t^{1 / z}\right)$,

where the local roughness exponent is $\chi_{l o c}=\chi-\theta$, $\beta_{*}=\theta / z=\beta-\chi_{l o c} / z$, and the scaling function $g_{A}(u)$ is not constant anymore for small arguments, but behaves as

$$
g_{A}(u) \sim\left\{\begin{array}{lll}
u^{-2\left(\chi-\chi_{l o c}\right)} & \text { if } & u \ll 1 \\
u^{-2 \chi} & \text { if } & u \gg 1
\end{array} .\right.
$$

The fact that $\theta \neq 0$ in Eq. (15) yields a local roughness exponent $\chi_{l o c} \neq \chi$ and an anomalous growth exponent $\beta_{*} \neq 0$. Therefore, there are now three independent exponents describing the scaling properties of the surface, whereas for Family-Vicsek scaling (even in the presence of super-roughening) there are only two. Which triplet of independent exponents one considers depends mainly on the kind of scaling properties one is measuring. In experiments normally it is local properties of the surface (local width, etc.) that are measured, and one might naturally consider as independent exponents e.g. $\chi_{l o c}, z$, and $\beta_{*}$. On the other hand, the focus of numerical simulations are usually finite size effects, thus probing global properties of the surface such as the global width or power spectrum. In the latter cases a common choice is e.g. $\chi, \theta$, and $\beta$. We should stress that, although the scaling relations for exponents are exactly the same as for the super-roughening case discussed above, the present type of anomalous scaling is due to $\theta \neq 0$ and may and does occur in growth models with $\chi<1$. The scaling behavior (18) of the local width is formally equivalent to that of super-roughening (for which case $\chi_{l o c}=1$ and $\left.\beta_{*}=\beta-1 / z\right)$, a fact which has produced some confusion in previous works where both anomalies have been identified in some way.

In view of the local scaling, Eq. (18), the structure factor can be rewritten conveniently as

$$
S(k, t) \sim\left\{\begin{array}{ll}
t^{(2 \chi+1) / z} & \text { if } k t^{1 / z} \ll 1 \\
k^{-\left(2 \chi_{l o c}+1\right)} t^{2\left(\chi-\chi_{l o c}\right) / z} & \text { if } k t^{1 / z} \gg 1
\end{array},\right.
$$

where we can observe two interesting facts that characterize what we call an intrinsic anomalous scaling. First, $S\left(k, t^{1 / z} \gg k^{-1}\right)$ decays as $k^{-\left(2 \chi_{l o c}+1\right)}$, and not following the $k^{-(2 \chi+1)}$ law characteristic of Family-Vicsek scaling (super-roughening case included). Second, there is an unconventional dependence of $S\left(k, t^{1 / z} \gg k^{-1}\right)$ on time which leads to a non stationary structure factor for $t^{1 / z} k \gg 1$. The combination of these two facts allows to distinguish between anomalous scaling due to superroughening $(\chi>1)$ and intrisic anomalous scaling.

\section{MODELS AND NUMERICAL RESULTS}

In this section we present numerical simulations of two different growth equations in order to illustrate the theory we have just discussed in the previous section. First, we discuss the linear MBE equation as a paradigmatic example of Family-Vicsek behavior of the structure factor, in the presence of super-roughening, leading to anomalous scaling of the height-height correlation function. Second, we study a random diffusion model in which one can tune the values of the global exponents $\chi, \beta$. We compare the exact solution of the model with numerical integrations of the equation of motion. By analyzing the structure factor, we will see that the second model constitutes an excellent example of what we have termed intrinsic anomalous scaling in the previous section, independent of the value of $\chi$ below or above one.

\section{A. Linear MBE equation: super-roughening}

Let us start studying the simplest equation relevant for MBE growth [18,8,9]. For a one dimensional interface it reads

$$
\frac{\partial h}{\partial t}=-K \frac{\partial^{4} h}{\partial x^{4}}+\eta(x, t)
$$

where $\eta$ is a white noise with zero mean, and the constant $K>0$. Exponents can be easily found by dimensional analysis, $\beta=3 / 8, z=4$, and $\chi=3 / 2$, thus Eq. (21) generates super-rough surfaces. This equation has been widely studied by many authors; however for the sake of clarity we wish to sumarize here briefly some numerical results for it that illustrate our scaling analysis in an example of super-roughening.

We have solved numerically Eq. (21) by means of a finite differences scheme. To study the structure factor, we have calculated $S(k, t)$ in systems of $L=$ $16,32,64,128,256$. 


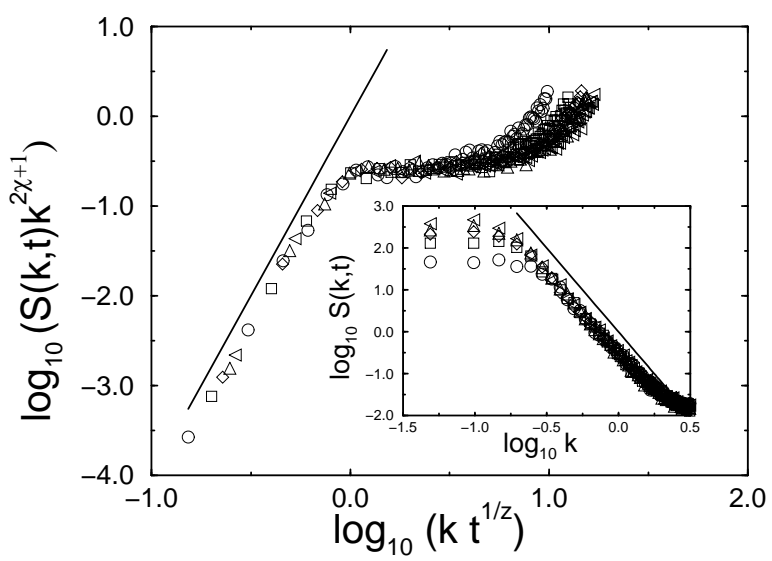

FIG. 1. The inset shows the structure factor for the linear MBE equation, Eq.(21), calculated in a system of total size $L=128$ for times $t=10^{2}, 3 \times 10^{2}, 5 \times 10^{2}, 7 \times 10^{2}, 9 \times 10^{2}$. The solid straight line has slope -4 and is ploted to guide the eye. In the main panel the data are collapsed using exponents $\chi=3 / 2, z=4$. The solid line has slope 4 , showing that the scaling function of the structure factor is consistent with Eq. (10).

In the inset of Fig. 1 numerical results are shown for $L=128$, and 200 realizations of the noise. For $k^{-1} \gg t^{1 / z}$, the structure factor decays as $k^{-4}$ (i. e. the global roughness exponent $\chi=3 / 2$ ) and does not display any anomaly. In Fig. 1 we plot a collapse of the data for the exponents $z=4$ and $\chi=3 / 2$. As we see, the scaling function of the structure factor indeed has the Family-Vicsek shape (10) [19]. Thus there is no anomaly in the behavior of $S(k, t)$ for Eq. (21). However, there is anomalous behavior if we look at the local properties of the surface in real space. We have calculated the local width, $w(l, t)$ for different window sizes $l$ in a system of total size $L=1000$, and averaged over 15 realizations of the noise.

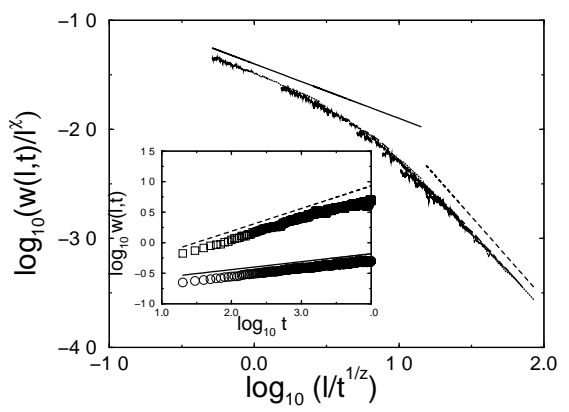

FIG. 2. Numerical results for the local width of the linear MBE equation in a system of size $L=1000$. In the inset we plot $w(l, t)$ vs. time for two different window sizes $l=5$ (०) and $l=150(\square)$. The straight lines have slopes 0.13 (solid) and 0.37 (dashed), corresponding to $\beta_{*}$ and $\beta$ respectively. Data are collapsed in the main panel for $l=5$, up to $l=150$ using exponents $\chi=3 / 2, z=4$. The straight lines correspond to $u^{-\chi}$ (dashed) and $u^{-\left(\chi-\chi_{l o c}\right)}$ (solid) and have slopes $-3 / 2$ and $-1 / 2$ respectively.
An inspection of the inset of Fig. 2 shows that the local width on length scales $l \ll L$ does not saturate at times $t \gg l^{z}$ but continues to grow in time as $w\left(l, t \gg l^{z}\right) \sim t^{\beta_{*}}$ with $\beta_{*} \simeq 0.13$, in agreement with the theoretical prediction (14) for Eq. (21) $\beta_{*}=\beta-1 / z=0.125$.

As discussed above, in the presence of anomalous scaling the height-height correlation function (or the local width) satisfies a dynamic scaling form, although with a scaling function which differs from the Family-Vicsek one, see Eqs. (13), (18), and (19). As stressed in 11] (for the height-height correlation function), the plot of $w(l, t) / l^{\chi}$ vs. $l / t^{1 / z}$ allows one to determine the form of the scaling function since

$$
\frac{w(l, t)}{l \chi} \sim g\left(l / t^{1 / z}\right)
$$

The data in the inset of Fig. 2 have been collapsed for $l=5, \ldots, 150$, and plotted in Fig. 2. The scaling function $g(u)$ obtained is not constant for $u \ll 1$, but scales with its argument as $u^{-\left(\chi-\chi_{l o c}\right)}$, with $\chi_{l o c}=1$ for this case. This is consistent with the scaling behaviour (14), or equivalently with (18) for $\chi_{l o c}=1$.

\section{B. Random diffusion model: Intrinsic anomalous scaling}

Next let us consider the more complex case of intrinsic anomalous scaling. We focus our attention on a growth model with a random diffusion coefficient governed by the equation

$$
\frac{\partial h(x, t)}{\partial t}=\frac{\partial}{\partial x}\left(D(x) \frac{\partial}{\partial x} h(x, t)\right)+\eta(x, t) .
$$

The diffusion coefficient $D(x)>0$ is distributed according to the probability density $P(D)=N_{\phi} D^{-\phi} f_{c}\left(D / D_{c}\right)$, where the parameter $\phi$ characterizes the intensity of the disorder. $N_{\phi}$ is merely a normalization constant and the cutoff function is $f_{c}(y)=1$ for $y \leq 1$ and $f_{c}(y)=0$ for $y>1$. If $\phi<0$, disorder in the diffusion coefficient does not play any role and the Edwards-Wilkinson [6] exponents $\chi=\chi_{l o c}=1 / 2, \beta=1 / 4$ are recovered. Thus the disorder is termed weak. On the contrary, for strong disorder, $0<\phi<1$, the critical exponents $\chi, \beta$ are disorder-dependent (through the value of $\phi$ ).

There are two major features that make this model interesting. On one hand, it is linear and can be solved exactly 13,12$]$. As a consequence, exponents are dependent on the parameter $\phi$ in a known way. In particular, the global roughness exponent $\chi$ takes a continuous range of values from $\chi=1 / 2$ to $\chi=\infty$ when varying the intensity of disorder from $\phi=0$ to 1 . In Ref. 12 it has been found that the random diffusion model always exhibits anomalous scaling for $0<\phi<1$. Hence anomalous scaling in this model occurs for surfaces with roughness exponent $\chi>1$ as well as for those with $\chi<1$. 
Therefore, this model constitutes an excellent example of anomalous scaling not due to super-roughening.

As stated above, the analytical study of (23) allows to determine exactly the critical exponents (see [12]). For the region of interest here, $0<\phi<1$, one has

$$
\beta=\frac{1}{2(2-\phi)}, \quad \chi=\frac{1}{2(1-\phi)}, \quad \chi_{l o c}=\frac{1}{2}
$$

It is a curious property of model (23) that $\chi_{l o c}$ remains constant when changing the intensity of the disorder, in contrast to the wide range of variation existing in $\chi$. This is a feature also found for many models of rough epitaxial growth 11]. In Sec. II we have demonstrated that in the case of anomalous kinetic roughening the time exponent $\beta_{*}$ is always given by the scaling relation $\beta_{*}=\beta-\chi_{\text {loc }} / z$. Thus, from (24), $\beta_{*}$ depends on $\phi$ as $\beta_{*}=\phi /(4-2 \phi)$ for a given disorder parameter $\phi$.

We have performed simulations of the discretized version of Eq. (23) in a system of lateral size $L=1000$ for different degrees of disorder in the strong disorder phase, $0<\phi<1$. In Fig. 3 we plot $w(l, t)$ for two values of $\phi$. For $\phi=2 / 3$, the exact global roughness exponent is larger than one, $\chi=3 / 2$, and one observes in the upper panel of Fig. 3 that the scaling behaviour of the local width is anomalous with $\beta_{*}=0.27 \pm 0.03$ in agreement with the theoretical value $\beta_{*}=1 / 4$.

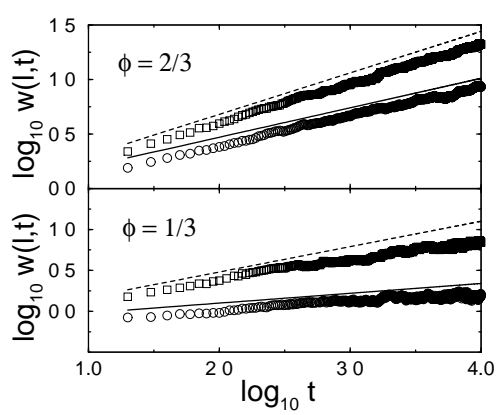

FIG. 3. Local width vs. time for the random diffusion model, Eq. (23), calculated over windows of size $l=5$ (०) and $l=150(\square)$ for two different degrees of disorder. In the upper panel $(\phi=2 / 3)$, the straight lines have slopes 0.27 (solid) and 0.38 (dashed), to be compared with the exact values $\beta_{*}=1 / 4$ and $\beta=3 / 8$, respectively. In the lower panel $(\phi=1 / 3)$, the lines have slopes 0.12 (solid) and 0.31 (dashed), to be compared with $\beta_{*}=1 / 10$ and $\beta=3 / 10$, respectively. Thus, the scaling of model (23) is anomalous (i.e. $\beta_{*} \neq 0$ ) independently of the value of the global roughenss exponent.

In the case of a disorder parameter $\phi=1 / 3$ (see lower panel of Fig. 3), for which $\chi=3 / 4<1$, the scaling of $w(l, t)$ is also anomalous with an anomalous time exponent $\beta_{*}=0.12 \pm 0.04$ to be compared to $\beta_{*}=1 / 10$ predicted by the exact analysis. Thus, in equation (23) the existence of anomalous scaling is completely independent of the surface being super-rough or not.

As we did above for the linear MBE equation, we have determined the scaling function $g(u)$ of the local width by the data collapse shown in Fig. 4, in which the exponents obtained from Fig. 3 have been used. For both degrees of disorder $\phi=1 / 3$ and $2 / 3$, the scaling function behaves as expected for anomalous scaling, and has an analogous shape to that found for the local width in the super-rough case.

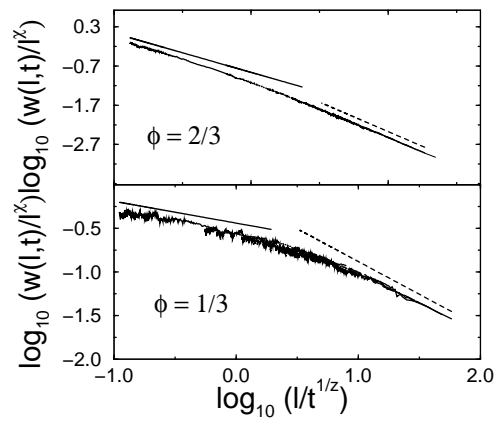

FIG. 4. Data collapse of the results displayed in Fig. 3. In the upper panel $(\phi=2 / 3)$, the exponents $\chi=3 / 2, z=4.08$ have been used. The straight lines are plotted as a guide to the eye having slopes $-3 / 2$ (dashed) and -1 (solid). For $\phi=1 / 3$ (lower panel), the data show a good collapse for $\chi=3 / 4, z=2.42$. The straight lines have slopes -0.25 (solid) and -0.75 (dashed). In both panels the scaling agrees with formula (25).

The complete scaling function $g(u)$ defined in (22) is thus given by

$$
g(u) \sim\left\{\begin{array}{ll}
u^{-\left(\chi-\chi_{l o c}\right)} & \text { if } u \ll 1 \\
u^{-\chi} & \text { if } u \gg 1
\end{array},\right.
$$

where $\chi$ is the disorder-dependent global roughness exponent given in (24) and $\chi_{l o c}=1 / 2$.

The intrisic anomalous character of the scaling in this model can be diagnosed through the use of the structure factor. We have calculated $S(k, t)$ in systems of sizes $L=16, \ldots, 512$. Figure 5 shows our results for $L=128$, and 200 realizations of the disorder.

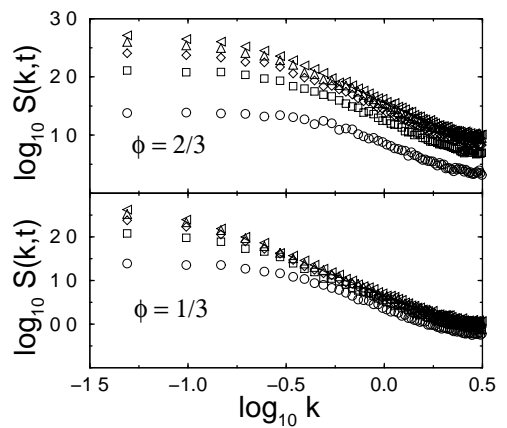

FIG. 5. Structure factor of the random diffusion model Eq. (23) for two different degrees of the disorder and times $t=10^{2}, 3 \times 10^{2}, 5 \times 10^{2}, 7 \times 10^{2}, 9 \times 10^{2}$. Upper panel, results for $\phi=2 / 3(\chi=3 / 2)$. Lower panel, data for $\phi=1 / 3$ $(\chi=3 / 4)$. The shift in time reflects the intrinsic anomalous character of the scaling.

For both values of the disorder parameter $\phi=1 / 3,2 / 3$ the spectrum decays as $k^{-\left(2 \chi_{l o c}+1\right)}\left(\right.$ not as $\left.k^{-(2 \chi+1)}\right)$ and 
is clearly shifted for different times. This scaling behaviour is the one in Eq. (20), which we associated in Sec. II.B with that of growth models having an intrisic anomaly. This can be better appreciated when collapsing the curves of Fig. 5 as shown in Fig. 6, which displays the $S(k, t)$ data collapses for $\phi=1 / 3,2 / 3$, and yields a scaling function $s(u)$ with a form consistent with Eq. (15), and not with the Family-Vicsek form, Eq. (10) [19].

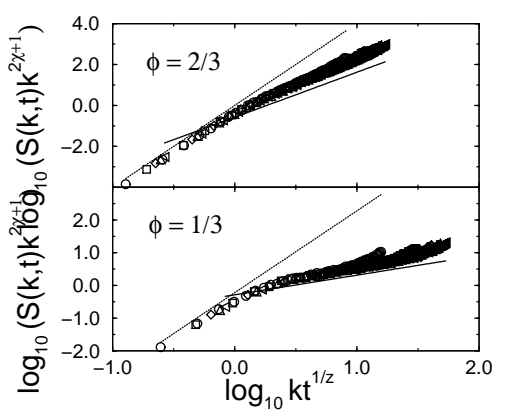

FIG. 6. Data collapse of the graphs in Fig. 5. The exponents used for the collapse in the upper panel $(\phi=2 / 3)$ are $\chi=3 / 2$ and $z=4.08$. The straight lines have slopes 2.2 (solid) and 4 (dashed). In the lower panel $(\phi=1 / 3)$ the exponents used for the collapse are $\chi=3 / 4$ and $z=2.42$. The straight lines have slopes 0.6 (solid) and 2.5 (dashed). In both panels the scaling function is described by Eq. (15).

As we see, this example clearly shows that intrinsic anomalous behaviour may occur in the case of a roughness exponent $\chi=3 / 2>1(\phi=2 / 3)$ as well as for $\chi=3 / 4<1(\phi=1 / 3)$.

\section{CONCLUSIONS}

As we have seen in the previous sections, one of the main conclusions of the present work is that anomalous scaling of rough surfaces is a more general (and independent) phenomenon than those cases associated with super-roughening. In this sense several issues associated with anomalus scaling remain to be clarified. One of them is its relation and/or interplay with the phenomena of multifractality and intermitency, known to take place in the super-rough case 20,21]. Moreover, we have seen that the intrinsic anomalous surfaces display a novel type of spectrum thus far not considered for self-affine geometries, which are usually defined as to have a FamilyVicsek spectrum 22]. An outstanding issue in this regard is to clarify the physical meaning of the exponent $\theta$, as well as extending the currently available renormalization group techniques to be able to calculate the value of this exponent. On the phenomenological side, when performing experiments or interpreting numerical simulations of discrete growth models, we believe the existence of intrinsic anomalous scaling is a very relevant issue. On one hand, in the presence of intrinsic anomalous scaling the various correlation functions behave somewhat sim- ilarly to the Family-Vicsek case. However, indeed there are important differences. For instance, $S(k, t)$ scales as $k^{-\left(2 \chi_{l o c}+1\right)}$, and not as $k^{-(2 \chi+1)}$, the graphs of $w(l, t)$ and $S(k, t)$ are shifted for different times, etc. There do exist data collapses, but again they are different from the usual Family-Vicsek type. For instance, if instead of plotting $w(l, t) / l^{\chi}$ vs. $l / t^{1 / z}$ as recommended in [11] and done here in Figs. 2,4 , one chooses to represent $w(l, t) / t^{\beta}$ vs. $l / t^{1 / z}$ as is frequently done, one gets, in the Family-Vicsek case,

$$
\frac{w(l, t)}{t^{\beta}} \sim\left\{\begin{array}{ll}
\text { const. } & \text { if } l / t^{1 / z} \ll 1 \\
\left(\frac{l}{t^{1 / z}}\right)^{\chi} & \text { if } l / t^{1 / z} \gg 1
\end{array},\right.
$$

whereas for intrinsic anomalous scaling one gets

$$
\frac{w(l, t)}{t^{\beta}} \sim\left\{\begin{array}{ll}
\text { const. } & \text { if } l / t^{1 / z} \ll 1 \\
\left(\frac{l}{t^{1 / z}}\right)^{\chi-\theta}=\left(\frac{l}{t^{1 / z}}\right)^{\chi_{l o c}} & \text { if } l / t^{1 / z} \gg 1
\end{array},\right.
$$

that is, in both cases there is data collapse with a very similar shape of the scaling function. However, the slopes of the corresponding scaling functions for large arguments are different. In the Family-Vicsek case, the slope coincides with the value of the exponent assumed to achieve the collapse of the data. However, in the anomalous case it does not. Therefore, it is crucial to check whether the slope of the scaling function does or not coincide with the assumed exponents. We believe this has not always been done when analyzing data from experiments and/or numerical simulations, and may have added to certain confusion existing in the literature in the identification of universality classes. Moreover, the intermediate time regime existing in surfaces with anomalous scaling introduces difficulties in the evaluation of exponents through the common use of local measurements such as the local width, frequently employed in experiments and numerical simulations. This may lead to the assignment of erroneous effective values (see [12]) to the exponents of surfaces hypothesized to behave in the simple FamilyVicsek fashion, while a more accurate description of their true scaling properties may come through the use of the anomalous scaling Ansatz.

\section{ACKNOWLEDGMENTS}

We thank E. Moro and A. Sánchez for discussions and encouragement. J. M. L. acknowledges the Postdoctoral Pogram of Universidad de Cantabria for support at Instituto de Física de Cantabria where most of this work has been made. R. C. also acknowledges warm hospitality at Instituto de Física de Cantabria. This work has been supported by DGICyT of the Spanish Government under Project Nr. PB93-0054-C02-02. 


\section{APPENDIX A: LOCAL SCALING PROPERTIES OF SURFACES}

In this Appendix we study the scaling properties of the height-height correlation function $G(l, t)$ from the different behaviors of the structure factor $S(k, t)$. In all cases, the starting point is the general formula

$$
\begin{aligned}
G(l, t) & =\int_{\frac{2 \pi}{L}}^{\frac{\pi}{a}}[1-\cos (k l)] \frac{s\left(k t^{1 / z}\right)}{k^{2 \chi+1}} d k \\
& =l^{2 \chi} \int_{\frac{2 \pi l}{L}}^{\frac{\pi l}{a}}[1-\cos (u)] \frac{s(\rho u)}{u^{2 \chi+1}} d u,
\end{aligned}
$$

where $s(\cdot)$ is the corresponding scaling function of the structure factor under study, and $\rho=t^{1 / z} / l$.

\section{Saturation regime, $l \ll L \ll t^{1 / z}$.}

In this regime, $\rho \gg 1$, and the smallest possible value of the argument of $s(\rho u)$ in A1 is also $\rho \pi l / L \sim t^{1 / z} / L \gg$ 1. Therefore we can replace the scaling function $s(\rho u)$ by its behavior at large arguments, to get the following results:

Family-Vicsek with $\chi<1$ : In this case the scaling function $s(\rho u)=s_{F V}(\rho u)$ of Eq. (10). We get

$$
G(l, t) \sim l^{2 \chi} \int_{0}^{\infty} \frac{1-\cos u}{u^{2 \chi+1}} d u \sim l^{2 \chi},
$$

where the limits $L \rightarrow \infty$ and $a \rightarrow 0$ can be taken, and the integral yields a finite constant.

Family-Vicsek with $\chi \geq 1$ : In this case the scaling function is as above $s(\rho u)=s_{F V}(\rho u)$. However, for $\chi>1$ there is an ultraviolet divergence in the integral (A2). We can avoid the divergence by keeping finite the lower integration limit (i.e. taking the limits $a \rightarrow 0, t^{1 / z} / L \rightarrow \infty$ for a fixed $L$ ). In this way we have

$$
\begin{aligned}
G(l, t) & \sim l^{2 \chi} \int_{2 \pi l / L}^{\infty} \frac{1-\cos u}{u^{2 \chi+1}} d u \sim l^{2 \chi}\left(\frac{l}{L}\right)^{2(1-\chi)} \\
& =l^{2} L^{2(\chi-1)}
\end{aligned}
$$

where we have kept only the most singular term (as can be shown integrating by parts in the above formula). As stated in the text, this implies a local value for the roughness exponent $\chi_{l o c}=1$. The case $\chi=1$ is a marginal situation. Following the same steps as in Eq. (A3), one obtains

$$
G(l, t) \sim l^{2 \chi} \log L
$$

that is, $\chi_{l o c}=\chi=1$ up to logarithmic corrections.

Intrinsic anomalous scaling: Now the scaling function we have to use in Eq. (A1) is $s(\rho u)=s_{A}(\rho u) \sim(\rho u)^{2 \theta}$, see (15). Thus

$$
G(l, t)=l^{2 \chi} \rho^{2 \theta} \int_{0}^{\infty} \frac{1-\cos u}{u^{2 \chi+1-2 \theta}} d u .
$$

The integrals are convergent as long as $\theta \equiv \chi-\chi_{l o c}>0$ and $\chi_{l o c}<1$ (the latter being always fulfilled as shown in 15, 16]). At saturation we thus have

$$
G(l, t) \sim l^{2(\chi-\theta)} L^{2 \theta}=l^{2 \chi_{l o c}} L^{2 \theta},
$$

hence the difference between local and global scaling behavior (note, however, $G(l \sim L, t) \sim L^{2\left(\theta+\chi_{l o c}\right)}=L^{2 \chi}$ ).

\section{Early dynamics, $t^{1 / z} \ll l \ll L$.}

In this case the minimum value of the argument of the scaling function in (A1) is $\rho \pi l / L \sim t^{1 / z} / L \ll 1$, so that in principle the integral probes the whole scaling function $s(\rho u)$, for both small and large values of its argument. In this subsection we first consider the case in which, further, we have $\rho=t^{1 / z} / l \ll 1$. Let us perform a change of variables in expression (A1):

$$
G(l, t)=l^{2 \chi} \rho^{2 \chi} \int_{2 \pi t^{1 / z} / L}^{\pi t^{1 / z} / a}\left[1-\cos \left(\frac{y}{\rho}\right)\right] \frac{s(y)}{y^{2 \chi+1}} d y .
$$

We want to study the asymptotics of the integral in (A7) in the limit $\rho \rightarrow 0$. Using standard techniques (see $e . g$. [17]), and assuming $\lim _{y \rightarrow 0} s(y)<\infty$, it is easy to show that, for $\rho \rightarrow 0$,

$$
\int_{2 \pi t^{1 / z} / L}^{\pi t^{1 / z} / a}\left[1-\cos \left(\frac{y}{\rho}\right)\right] \sim \int_{0}^{\infty} \frac{s(y)}{y^{2 \chi+1}} d y+\mathcal{O}(\rho)
$$

where the integral appearing in the last equation is well behaved in its limits, and $s(y)$ is any of the scaling functions we are considering, either $s_{F V}(y)$ or $s_{A}(y)$. Therefore we get

$$
G(l, t) \sim l^{2 \chi} \rho^{2 \chi}+\mathcal{O}\left(\rho^{2 \chi+1}\right) \sim t^{2 \chi / z} \text { for } t^{1 / z} \ll l,
$$

that is, the scaling properties of the height-height correlation function in the early dynamics are the same irrespective of the scaling behavior of the structure factor, whether Family-Vicsek or intrinsically anomalous.

\section{Intermediate time regime, $l \ll t^{1 / z} \ll L$.}

Finally, we study the case in which, analogously to the previous subsection, the minimum value for the argument of the scaling function along the integration region in (A1) is small — so that the whole scaling function contributes in principle - but the parameter $\rho=t^{1 / z} / l$ is large. That is, we are in the intermediate time regime. 
In this case, again we have to distinguish between FV and intrinsic anomalous scaling of $S(k, t)$.

Family-Vicsek scaling with $\chi<1$ : When $s(\rho u)=$ $s_{F V}(\rho u)$ in Eq. (A1), the limits $L \rightarrow \infty, a \rightarrow 0$ can be taken after $\rho \rightarrow \infty$, and

$$
G(l, t) \sim l^{2 \chi} \int_{0}^{\infty}(1-\cos u) \frac{1}{u^{2 \chi+1}} d u \sim l^{2 \chi},
$$

being the integral a finite constant.

Family-Vicsek scaling with $\chi \geq 1$ : Again for the case of super-roughening with FV scaling in $S(k, t)$ an expansion such as that in Eq. A10) leads to a divergent integral. The behavior of $G(l, t)$ is best analyzed in the form (A7). For $\chi>1$, we can take the limits $t^{1 / z} / a \rightarrow \infty$, $t^{1 / z} / L \rightarrow 0$ to get

$$
G(l, t) \sim l^{2 \chi} \rho^{2 \chi-2} \int_{0}^{\infty} \frac{s_{F V}(y)}{u^{y \chi+1}} \sim l^{2} t^{2(\chi-1) / z} .
$$

In the marginal case $\chi=1$, the integral in the above expression diverges when taking the mentioned limits $t^{1 / z} / a \rightarrow \infty, t^{1 / z} / L \rightarrow 0$, being the most divergent term logarithmic in time, that is:

$$
G(l, t) \sim l^{2} \log t .
$$

Comparing with Eq. (14), this yields $\beta_{*}=0$ for $\chi=1$, meaning logarithmic corrections in time.

Intrinsic anomalous scaling. When we use $s(\rho u)=$ $s_{A}(\rho u)$ in (A1) and proceed as in (A10), the behavior of the scaling function at large arguments now contributes with a leading term (in the limit $\rho \rightarrow \infty$ for fixed $\rho l / a$ and $\rho l / L)$ of the form

$$
\begin{aligned}
G(l, t) & \sim l^{2 \chi} \rho^{2 \theta} \int_{0}^{\infty}(1-\cos u) \frac{1}{u^{2 \chi+1}} d u \sim l^{2(\chi-\theta)} t^{2 \theta / z} \\
& =l^{2 \chi_{l o c}} t^{2 \beta_{*}},
\end{aligned}
$$

hence the anomalous behavior of the height-height correlation function summarized in (18).

[1] A.-L. Barabási and H. E. Stanley, Fractal Concepts in Surface Growth (Cambridge University Press, Cambridge, 1995).
[2] J. Krug and H. Spohn, in Solids Far From Equilibrium, C. Godréche ed. (Cambridge University Press, Cambridge, 1991).

[3] P. Meakin, Phys. Rep. 235, 189 (1993).

[4] T. Halpin-Healey and Y.-C. Zhang, Phys. Rep. 254, 215 (1995).

[5] F. Family and T. Vicsek, J. Phys. A 18, L75 (1985).

[6] S. F. Edwards and D. R. Wilkinson, Proc. R. Soc. London A 381, 17 (1982).

[7] M. Kardar, G. Parisi and Y.-C. Zhang, Phys. Rev. Lett. 56, 889 (1986).

[8] J. Amar, P.-M. Lam, and F. Family, Phys. Rev. E 47, 3242 (1993).

[9] S. Das Sarma, S. V. Ghaisas, and J. M. Kim, Phys. Rev. E 49, 122 (1994).

[10] M. Plischke, J. D. Shore, M. Schroeder, M. Siegert, and D. E. Wolf, Phys. Rev. Lett. 71, 2509 (1993); M. Schroeder, M. Siegert, D. E. Wolf, J. D. Shore, and M. Plischke, Europhys. Lett. 24, 563 (1993).

[11] See a recent survey for various growth models in S. Das Sarma, C. J. Lanczycki, R. Kotlyar, and S. V. Ghaisas, Phys. Rev. E 53, 359 (1996).

[12] J. M. López and M. A. Rodríguez, Phys. Rev. E 54, R2189 (1996).

[13] J. M. López and M. A. Rodríguez, Phys. Rev. E 52, 6442 (1995).

[14] Incidentally, it has been argued that $S(k, t)$ provides in general the most reliable method to estimate exponents for self-affine objects, see J. Schmittbuhl, J.-P. Villote, and S. Roux, Phys. Rev. E 51, 131 (1995); in the context of kinetically roughened surfaces, M. Siegert, Phys. Rev. E 53, 3209 (1996).

[15] H. Leschhorn and L.-H. Tang, Phys. Rev. Lett. 70, 2973 (1993).

[16] S. Roux and A. Hansen, J. Phys. I France 4, 515 (1994).

[17] C. M. Bender and S. A. Orszag, Advanced Mathematical Methods for Scientists and Engineers (McGraw-Hill, Singapore, 1984).

[18] J. Villain, J. Phys. I France 1, 19 (1991).

[19] The curvature of the collapsed data at large values of the abscissa is an effect of the discrete lattice, see e.g. M. Siegert in 14].

[20] J. Krug, Phys. Rev. Lett. 72, 2907 (1994).

[21] S. Galluccio, private communication.

[22] K. Falconer, Fractal Geometry: Mathematical Foundations and Applications (John Wiley \& Sons, Chichester, 1993). 